\section{Autologous stem cell transplantation for progressive systemic sclerosis: a prospective non-interventional study from the European Society for Blood and Marrow Transplantation Autoimmune Disease Working Party}

\author{
Joerg Henes, ${ }^{1}$ Maria Carolina Oliveira, ${ }^{2}$ Myriam Labopin, ${ }^{3}$ Manuela Badoglio, ${ }^{4}$ \\ Hans Ulrich Scherer, ${ }^{5}$ Nicoletta Del Papa, ${ }^{6}$ Thomas Daikeler, ${ }^{7}$ Marc \\ Schmalzing, ${ }^{8}$ Roland Schroers, ${ }^{9}$ Thierry Martin, ${ }^{10}$ Gregory Pugnet, ${ }^{11}$ Belinda \\ Simoes, ${ }^{12}$ David Michonneau, ${ }^{13}$ Erik W.A. Marijt, ${ }^{14}$ Bruno Lioure,${ }^{15}$ Jacques \\ Olivier Bay, ${ }^{16}$ John A Snowden, ${ }^{17}$ Montserrat Rovira, ${ }^{18}$ Anne Huynh, ${ }^{19}$ Francesco \\ Onida, ${ }^{20}$ Lothar Kanz, ${ }^{21}$ Zora Marjanovic ${ }^{22}$ and Dominique Farge ${ }^{23}$ for the \\ NISSC1 members
}

${ }^{1}$ Center for Interdisciplinary Clinical Immunology, Rheumatology and Auto-inflammatory Diseases and Department of Internal Medicine II (Oncology, Hematology, Immunology and Rheumatology), University Hospital, Tübingen, Germany; ${ }^{2}$ Ribeirão Preto Medical School, University of São Paulo, Ribeirão Preto, Brazil; ${ }^{3} E B M T$ Paris Study Office, Saint Antoine Hospital, Université Pierre et Marie Curie, INSERM UMR 938, Department of Haematology, Paris, France; ${ }^{4}$ EBMT Paris Study Office, Hôpital St Antoine, INSERM UMR 938, Paris, France; ${ }^{2}$ Leiden University Medical Center, Department of Rheumatology, Leiden, the Netherlands; ${ }^{\circ}$ Scleroderma Clinic, Ospedale Pini, Department of Rheumatology, Milan, Italy; ${ }^{7}$ University and University Hospital of Basel, Department of Rheumatology, Basel, Switzerland; ' $U$ niversity Hospital of Würzburg, Department of Rheumatology/Immunology, Würzburg, Germany ${ }^{9}$ Universitäts Klinik of Bochum, Med. Klinik, Bochum, Germany; ${ }^{10}$ Hôpitaux Universitaires de Strasbourg, Service de Médecine Interne et Immunologie Clinique, Centre de Référence des Maladies Auto-Immunes Systémiques Rares Est-Sud-Ouest (RESO), Strasbourg, France; ${ }^{11} \mathrm{CHU}$ de Toulouse, Hôpital Purpan, Service de Médecine Interne, Toulouse, France; ${ }^{12}$ Ribeirão Preto Medical School, University of São Paulo, Division of Hematology, Ribeirão Preto, Brazil; ${ }^{13}$ Hôpital Saint Louis \& Université Paris 7, Denis Diderot, Department of Hematology, Paris, France; ${ }^{14}$ Leiden University Medical Center, Department of Hematology, Leiden, the Netherlands; ${ }^{15}$ Strasbourg University Hospital, Department of Hematology, Strasbourg, France; ${ }^{16} \mathrm{CHU}$ de Clermont Ferrand, Department of Hematology, Clermont Ferrand, France; ${ }^{17}$ Department of Haematology, Sheffield Teaching Hospitals NHS Foundation Trust, Sheffield, UK; ${ }^{18} \mathrm{Hospital}$ Clínic of Barcelona, Department of Hematology, Barcelona, Spain; ${ }^{19}$ UCT Oncopole, Department of Hematology, Toulouse, France; ${ }^{20}$ Fondazione IRCCS Ca Granda Ospedale Maggiore Policlinico, University of Milan, Milan, Italy; ${ }^{21}$ University Hospital Tübingen, Department of Internal Medicine II (Oncology, Haematology, Immunology and Rheumatology), Tübingen, Germany ${ }^{22}$ Saint Antoine Hospital, Department of Hematology, Paris, France; ${ }^{23}$ Hôpital Saint-Louis, Assistance Publique des Hôpitaux de Paris, Centre de Référence des Maladies AutoImmunes Systémiques Rares d'Ile-de-France, Filière 'FAI2R', Unité de Médecine Interne: Maladies Auto-immunes et Pathologie Vasculaire (UF 04), Université de Paris, EA 3518, Paris, France and ${ }^{24}$ Department of Medicine, McGill University, Montreal, Quebec, Canada

\section{ABSTRACT}

T hree randomized controlled trials in early severe systemic sclerosis demonstrated that autologous hematopoietic stem cell transplantation was superior to standard cyclophosphamide therapy. This European Society for Blood and Marrow Transplantation multicenter, prospective, non-interventional study was designed to further decipher efficacy and safety of this procedure for severe systemic sclerosis patients in real-life practice and to search for prognostic factors. All consecutive adult patients with systemic sclerosis undergoing a first autologous hematopoietic stem cell transplant between December 2012 and February 2016 were prospectively included in the study. The primary endpoint was progression-free survival. Secondary endpoints were overall survival, non-relapse mortality, response and incidence of progression. Eighty patients with systemic sclerosis were included. The median dura-
Haematologica 2021

Volume 106(2):375-383

\section{Correspondence:}

DOMINIQUE FARGE

dominique.farge-bancel@aphp.fr;

dominique.farge@mcgill.ca

Received: June 22, 2019.

Accepted: January 9, 2020.

Pre-published: January 16, 2020.

https://doi.org/10.3324/haematol.2019.230128

(C)2021 Ferrata Storti Foundation

Material published in Haematologica is covered by copyright. All rights are reserved to the Ferrata Storti Foundation. Use of published material is allowed under the following terms and conditions:

https://creativecommons.org/licenses/by-nc/4.0/legalcode. Copies of published material are allowed for personal or internal use. Sharing published material for non-commercial purposes is subject to the following conditions: https://creativecommons. org/licenses/by-nc/4.0/legalcode, sect. 3. Reproducing and sharing published material for commercial purposes is not allowed without permission in writing from the publisher. 
tion of the follow-up was 24 (range, 6-57) months after stem cell transplantation using cyclophosphamide plus antithymocyte globulin conditioning for all, with CD34+ selection in 35 patients. At 2 years, the progression-free survival rate was $81.8 \%$, the overall survival rate was $90 \%$, the response rate was $88.7 \%$ and the incidence of progression was $11.9 \%$. The 100 -day non-relapse mortality rate was $6.25 \%(n=5)$ with four deaths from cardiac events, including three due to cyclophosphamide toxicity. Modified Rodnan skin score and forced vital capacity improved with time $(P<0.001)$. By multivariate analysis, baseline skin score $>24$ and older age at transplantation were associated with lower progression-free survival (hazard ratios 3.32 and 1.77, respectively). CD34+-cell selection was associated with better response (hazard ratio 0.46 ). This study confirms the efficacy of autologous stem cell transplantation, using nonmyeloablative conditioning, in real-life practice for severe systemic sclerosis. Careful cardio-pulmonary assessment to identify organ involvement at the time of the patient's referral, reduced cyclophosphamide doses and $\mathrm{CD} 34^{+}$-cell selection may improve outcomes. The study was registered at ClinicalTrials.gov: NCT02516124.

\section{Introduction}

Systemic sclerosis $(\mathrm{SSc})$ is a rare systemic autoimmune connective tissue disease characterized by vasculopathy, immune dysregulation and progressive fibrosis, affecting primarily the skin, gastrointestinal tract, lungs, heart and kidneys. ${ }^{1}$ It is associated with high disease-related mortali$t^{2}$ and the main causes of death are related to cardiac, pulmonary and renal involvement..$^{2.5}$ Mortality rates in patients with SSc have remained higher than those in the general population (standardized mortality ratio above 3.5) over the past decades, in contrast to reductions in mortality for other rheumatic diseases or cancer. ${ }^{6}$ After the diagnosis of non-Raynaud phenomenon, the following risk factors for higher mortality related to progressive disease have been identified:2 diffuse skin fibrosis measured by the modified Rodnan skin score (mRSS, range 0-51), elevated C-reactive protein levels, altered left or right ventricular ejection fraction, interstitial lung disease with reduced forced vital capacity (FVC) or diffusion capacity for carbon monoxide (DLCO) on lung function testing, proteinuria and male sex. Earlier studies had found that the presence of anti-topoisomerase II (Scl-70) auto-antibodies, reduced functional status - as measured by the Scleroderma Health Assessment Questionnaire (sHAQ, range from 0 to 3, with lower values indicating a better quality of life) $)^{8}$ - or pulmonary arterial hypertension adversely affect survival. ${ }^{3.5}$ Treatment with biologic or immunosuppressive drugs did not improve SSc survival. $2,9,10$

Following early phase I-II trials, ${ }^{11-13}$ three randomized controlled trials, namely ASSIST (American scleroderma stem cell versus immune suppression trial, phase II), ${ }^{14}$ ASTIS (Autologous stem cell transplantation international scleroderma trial, phase III) ${ }^{15}$ and SCOT (Scleroderma: cyclophosphamide or transplantation, phase II $)^{16}$ demonstrated the superiority of autologous hematopoietic stem cell transplantation (HSCT) compared to the standard cyclophosphamide pulse treatment with regards to overall and event-free survival. In this context, autologous HSCT has become the best treatment option for patients with severe or rapidly progressive SSc, with a grade 1 level of evidence since 2012 according to the Autoimmune Disease Working Party (ADWP) of the European Society for Blood and Marrow Transplantation (EBMT) recommendations. ${ }^{17,18}$ After two decades of progress in HSCT for autoimmune diseases, the use of autologous HSCT for severe or rapidly progressive SSc has also been endorsed by the European League Against Rheumatism (EULAR) ${ }^{10}$ and the American Society for Blood and Marrow Transplantation ${ }^{10,1,19}$ and the demand for this therapeutic procedure is the fastest growing indication for HSCT in Europe. . $^{10,1,19,20}$

We therefore designed this ADWP-EBMT non-interventional study, NISSC1, to further evaluate the efficacy and safety of autologous HSCT for early, severe, progressive SSc, when performed in real-life practice, and to identify risk factors for early adverse events and transplant-related mortality, in order to make this approach safer.

\section{Methods}

\section{Study design}

This study is a prospective, open, multicenter, non-interventional study. Data regarding patients included in this study were prospectively collected using a specific case report form, designed according to EBMT guidelines for HSCT in SSc. ${ }^{17}$ All EBMT participating centers agreed to report their consecutive patients aged 18 to 65 years with severe progressive SSc undergoing their first autologous HSCT between December 2012 and February 2016. Inclusion and exclusion criteria are detailed in the Online Supplementary Appendix. Participating centers followed their own local protocols for the transplant procedure as wells as the patients' evaluation at baseline and during the 2-year follow-up.

The study was approved by the EBMT board in 2012 and by local ethics committees in participating countries and registered at ClinicalTrials.gov (NCT02516124). All patients gave written informed consent to their participation in the study.

\section{Patients' evaluation}

The SSc patients' characteristics were assessed at baseline (within 3 months before autologous HSCT) and at 3, 6, 12, 18 and 24 months after transplantation (day 0 was defined as the time point of reinfusion of stem cells). Data collected included $\mathrm{mRSS}$, erythrocyte sedimentation rate (in $\mathrm{mm} / \mathrm{h}$ ), creatine kinase levels, presence or absence of autoantibodies, left ventricular ejection fraction (LVEF in \%) and systolic pulmonary arterial pressure (in $\mathrm{mmHg}$ ) on echocardiography, resting and $24 \mathrm{~h}$ Holter-electrocardiograms, abnormal cardiac magnetic resonance imaging (MRI) or pulmonary arterial hypertension on right heart catheterization (RHC), FVC and DLCO (\% of predicted values), high resolution computed tomography of the lungs and kidney function as well as immunosuppressive drugs given before transplantation. The sHAQ was used to evaluate patients' quality of life. 


\section{Autologous hematopoietic stem cell transplantation procedure}

Data collected included details on the mobilization process with or without $\mathrm{CD} 34^{+}$-cell selection, type of conditioning regimen; total dose of $\mathrm{CD}_{3} 4^{+}$cells infused; number of days of hospitalization for mobilization and conditioning, use and duration of granulocyte colony-stimulating factor (G-CSF), and time to engraftment.

\section{Endpoints}

The primary endpoint was progression-free survival (PFS), defined as survival after autologous HSCT without death or evidence of progression of SSc (see Online Supplementary Appendix S1).

The secondary endpoints were: (i) infectious or non-infectious adverse events during the first 100 days after autologous HSCT; (ii) engraftment, defined as 3 consecutive days of neutrophils $>0.5 \times 10^{9} / \mathrm{L}$ and platelets $>20 \times 10^{9} / \mathrm{L}$; (iii) response to treatment, defined as a $>25 \%$ improvement in $\mathrm{mRSS}$ and/or $\geq 10 \%$ improvement in FVC or DLCO as compared to baseline and without need of further immunosuppression; (iv) incidence of progression; (v) non-relapse mortality (NRM), defined as any death without progression; and (vi) overall survival.

\section{Statistical analysis}

Cumulative incidence functions were used to estimate response to treatment, NRM, and progression to accommodate for competing risks. Probabilities of overall survival and PFS were calculated using the Kaplan-Meier method. Univariate analyses were performed using the Gray test for cumulative incidence and the log rank test for overall survival and PFS. Multivariate analysis was performed using a Cox regression model including variables associated with the outcome with a $P$-value less than 0.10 in univariate analyses. Age was included as a continuous variable. A generalized linear model was used to analyze evolution over time of mRSS, FVC, DLCO and sHAQ. Results are expressed as a hazard ratio (HR) with $95 \%$ confidence interval (95\% CI).

\section{Results}

Eighty patients (71.3\% female), who had undergone a first autologous HSCT between December 2012 and February 2016 in 13 EBMT centers from six European countries and Brazil were included in this study. The centers are listed in Online Supplementary Appendix S2. The median (range) follow-up after transplantation was 24 (6-57) months. Table 1 summarizes the patients' baseline characteristics. The median disease duration since the diagnosis of SSc was 23.8 (5.3103.7) months.

At baseline, all 80 patients had skin involvement, and mRSS was above 15 in 64 (80\%) of them. Abnormal chest

Right heart catheterization, done

\section{Parameter} medlan (range)

\section{Patients}

80

\begin{tabular}{lc} 
Age at aHSCT, years & $43(20-62)$ \\
\hline Sex, female & $57(71.3)$ \\
Disease duration from SSc diagnosis, months & $23.8(5.3-103.7)$
\end{tabular}

Disease duration from SSc diagnosis, months

Body mass index (BMI)

$\mathrm{BMI}<18.5$

BMI 18.5-30

$8(10.3)$

BMI $>30$

\section{Skin involvement}

Modified Rodnan skin score (mRSS)

$24(2-49)$

Lung involvement

Lung crepitations

Pulmonary function tests, done

FVC, \% of predicted value

FEV1, \% of predicted value

DLCO, $\%$ of predicted value

Chest imaging, done

Chest X-rays, abnormal

Thoracic computed tomography (HRCT), abnormal*

Cardiac involvement

Systolic blood pressure (mmHg)

Diastolic blood pressure (mmHg)

Heart rate

Resting ECG, abnormal

24h Holter ECG, abnormal

Echocardiography, done

LVEF (in \%)

Pericardial effusion, present

sPAP by cardiac echo (mmHg)

Cardiac MRI, done

Cardiac MRI, abnormal**
41 (51.3)

$80(100)$

$72(43-132)$

$78(52-153.3)$

$59(34.3-120)$

$80(100)$

$31 / 71(43.7)$

$66 / 77$ (85.7)

$110(76-145)$

$70(40-90)$

$82(50-105)$

$4 / 80(5)$

$12 / 60(20)$

$80(100)$

$65(47-84)$

$5 / 79(6.3)$

$29(8-59)$

$59 / 80(74)$

$11(18.6)$
PAP (mmHg)

PAP, systolic

PAP, diastolic

$17 / 80(21.3)$

$17(8-23)$

$26(16-33)$

$10(6-16)$

$7(41)$

Musculo-skeletal involvement

Joints involvement

$42(52.5)$

Muscle weakness

$22(27.5)$

CK levels, U/L

Patients with CK level $\geq 250 \mathrm{U} / \mathrm{mL}$

$80(16-1368)$

9 (12.5)

Kidney function

Creatinine clearance, $\mathrm{mL} / \mathrm{min}^{* * *}$

Proteinuria on labstix

$111(58-190)$

Haematuria on labstix

9 (12)

7 (9.2)

SSc auto-antibodies

Anti-nuclear antibody positive

$74(92.7)$

Anti-topoisomerase I (Scl70) positive

$57(71.3)$

Anti-centromere positive

$3(4.2)$

Previous immunosuppressive SSc medication, yes**** $76(95.0)$

Cyclophosphamide (i.v.oral)

Dose, $g$

Methotrexate

$48(60)$

$6(1-17)$

Mycophenolate mofetil

Prednisone or equivalent

$46(58.2)$

Azathioprine

$16(20.3)$

$64(81.0)$

$8(10.0)$

Cyclosporine

$2(2.5)$

$3(3.8)$

If not otherwise stated all data are n (\%) or median (range) *Abnormal computed tomography scan includes fibrosis, honeycombing or ground-glass pattern. ${ }^{*}$ Abnormal cardiac magnetic resonance imaging includes reduced left or right ventricular function or late enhancement. ***The Cockcroft-Gault formula was recommended for estimating creatinine clearance, i.e. creatine clearance $=(140-$ age in years $) /$ serum creatinine in $\mathrm{mg} / \mathrm{dL}) \mathrm{x}$ body weight in $\mathrm{kg} / 72$ **** Previous treatment ever before, multiple answers possible. aHSCT: autologous hematopoietic stem cell transplantation; SSc: systemic sclerosis; BMI: body mass index; FVC: forced vital capacity; FEV1: forced experiratory volume in $1 \mathrm{~s}$; DLCO: diffusing capacity of lung for carbon monoxide; HRCT: high-resolution computed tomography; ECG: electrocardiography; LVEF: left ventricular ejection fraction; sPAP: systolic pulmonary artery pressure measured by echo echo: echocardiography; MRI: magnetic resonance imaging; PAP: pulmonary artery pressure; CK: creatine kinase; SSc: systemic sclerosis; ATG: antithymocyte globulins; 
x-rays or abnormal high-resolution computed tomography was reported in $70(87 \%)$ patients. Heart involvement with an abnormal 24 h electrocardiogram was reported in 12/60 $(20 \%)$ or with abnormal cardiac MRI in $11 / 59$ (19\%) patients. Among the 11 patients with abnormal MRI at baseline, echocardiography showed normal LVEF in all cases and pericardial effusion in one patient, the resting ECG was abnormal in one patient and the $24 \mathrm{~h}$ ECG was abnormal in two patients; baseline RHC performed in six patients (with fluid challenge in 2 cases) was normal. Overall, 21 patients $(26 \%)$ were transplanted without any prior cardiac MRI or RHC at baseline; among these, two had pericardial effusion and seven had a systolic pulmonary artery pressure above 25 $\mathrm{mmHg}$ on echocardiography, one had ventricular disturbances on resting ECG ( $\mathrm{n}=20)$, and 2/8 had an abnormal 24 h ECG.

\section{Mobilization and conditioning procedures}

All patients were mobilized using cyclophosphamide with a median dose of $2 \mathrm{~g} / \mathrm{m}^{2}$ (range, $1-4 \mathrm{~g} / \mathrm{m}^{2}$ ) plus G-CSF. The median duration of hospitalization was 4 (1-30) days for mobilization with large variation according to centers. CD34+-cell selection was performed in $35(44 \%)$ patients (Table 2).

The median time from mobilization to the start of the conditioning regimen for autologous HSCT was 52 (22-254) days. The conditioning regimen included cyclophosphamide in all 80 patients, alone in $76(95 \%)$ patients, with a median total dose of $200(50-240) \mathrm{mg} / \mathrm{kg}$. Four patients $(5 \%)$ received thiotepa $10 \mathrm{mg} / \mathrm{kg}$ with a total cyclophosphamide dose of $100 \mathrm{mg} / \mathrm{kg}$ as a single-center treatment for patients with cardiac abnormalities at the pre-transplant evaluation. Additional rabbit antithymocyte globulin (ATG), with different doses according to the brand, was also included in the conditioning regimen for all patients and administered over 4 to 5 days. Sixty-seven patients (82\%) received methylprednisolone during the ATG treatment. No patient received total body irradiation. A median number of $5.2 \mathrm{x}$ $10^{6} / \mathrm{kg}(2.53-23.31) \mathrm{CD}^{+} 4^{+}$cells were reinfused at transplantation. Following autologous HSCT, G-CSF was administered to $34(43 \%)$ patients for a median duration of $5(1-13)$ days. Following autologous HSCT, the median time to neutrophil engraftment was 11 (8-24) days and the median time to platelet engraftment was 9 (1-25) days. In 13 (16.9\%) patients, the platelet counts were never below $20 \times 10^{\circ} / \mathrm{L}$. There was an inverse correlation between the number of CD34 ${ }^{+}$cells infused and the time to engraftment (Spearman: $\left.r^{2}=-0.31 ; P<0.01\right)$, while the number of infused CD34+ cells and addition of G-CSF until recovery of aplasia were not associated with a reduced number of infectious complications $(P=0.32)$. The median duration of inpatient hospitalization for conditioning, autologous peripheral blood stem cell re-infusion and subsequent supportive care until engraftment and discharge was 24 (8-128) days (Table 2).

\section{Efficacy}

At 1 and 2 years after autologous HSCT, the PFS rate was 87.5\% (95\% CI: 80.2-94.7) and 81.8\% (95\% CI: 73.1-90.5), respectively, and the rate of response to treatment was $75 \%$ (95\% CI: 63.7-83.2) and 88.7\% (95\% CI: 79-94.1), respectively. At the 1- and 2-year time-points, the incidence of progression was $6.3 \%$ (95\% CI: 2.3-13.1) and $11.9 \%$ (95\% CI: $5.8-20.5)$, respectively, and the overall survival rate was 91.2\% (95\% CI: 85.1-97.4) and 90\% (95\% CI 83.4 - 96.6) at 1 and 2 years, respectively (Figure 1). Three of the four
Table 2. Treatment regimen used for mobilization and conditioning.

\begin{tabular}{|c|c|}
\hline Parameter & $\begin{array}{c}\mathrm{N}(\%) \text { or } \\
\text { median (range) }\end{array}$ \\
\hline $\begin{array}{l}\text { Mobilization } \\
\text { CYC } 2 \mathrm{~g} / \mathrm{m}^{2} \\
\text { CYC } 4 \mathrm{~g} / \mathrm{m}^{2} \\
\text { Other dose } \\
\text { Missing = } 2 \\
\text { G-CSF, yes } \\
\text { Leukapheresis } \\
1 \\
2 \\
3\end{array}$ & $\begin{array}{c}45(57.7 \%) \\
23(29.5 \%) \\
10(12.8 \%) \\
\\
80(100 \%) \\
61(77.2 \%) \\
11(13.9 \%) \\
7(8.9 \%)\end{array}$ \\
\hline $\begin{array}{l}\mathrm{CD}^{+} 4^{+} \text {selection } \\
\text { Yes } \\
\text { None }\end{array}$ & $\begin{array}{l}35(43.8 \%) \\
45(56.3 \%)\end{array}$ \\
\hline $\begin{array}{l}\text { Conditioning regimen } \\
\text { CYC } 200 \mathrm{mg} / \mathrm{kg} \\
\text { CYC other dose mg/kg } \\
\text { CYC } 100 \mathrm{mg} / \mathrm{kg}+\text { Thiotepa } 10 \mathrm{mg} / \mathrm{kg} \\
\text { Rabbit ATG, yes } \\
\text { Thymoglobulin (Sanofi/Genzyme), mg/kg } \\
\text { Grafalon (Neovi/Fresenius), mg/kg }\end{array}$ & $\begin{array}{c}72(90.0 \%) \\
4(5.0 \%) \\
4(5.0 \%) \\
80(100 \%) \\
7.5(2.5-7.5) \\
40(30-41)\end{array}$ \\
\hline $\begin{array}{l}\text { Growth factors } \\
\text { G-CSF administration after conditioning } \\
\text { N. of days of administration }\end{array}$ & $\begin{array}{c}34(43.0 \%) \\
5(1-13)\end{array}$ \\
\hline $\begin{array}{l}\text { Cells infused } \\
\text { CD } 34^{+} \text {cells reinfused, } 10^{6} / \mathrm{kg}\end{array}$ & $5.2(2.53-23.31)$ \\
\hline $\begin{array}{l}\text { Duration of hospitalization } \\
\text { Mobilization, days } \\
\text { Conditioning, days }\end{array}$ & $\begin{array}{c}4(1-30) \\
24(7-128)\end{array}$ \\
\hline
\end{tabular}

CYC: cyclophosphamide; G-CSF: granulocyte - colony-stimulating factor; ATG: antithymocyte globulin;

patients transplanted with thiotepa and a reduced-dose cyclophosphamide regimen responded; one with worsening skin and lung involvement at day 350 was then treated with prednisone, mycophenolate mofetil and rituximab. By multivariate analysis, mRSS $>24$ at baseline and older age at transplantation were significantly associated with a lower PFS with hazard ratios of 3.33 (95\% CI: 1.04-10.62) and 1.77 (95\% CI: 1.07-2.94), respectively. CD34+-cell selection was associated with a better response to therapy (HR: 0.46; 95\% CI: 0.27-0.76).

Figure 2 illustrates the evolution of the parameters over time. The mean mRSS decreased from 23.9 (standard deviation [SD] 9.7) at baseline to 14.2 (SD 9.2) at 1 year and 12.6 (SD 8.3) at 2 years $(n=55, P<0.001)$. Regarding lung function, the mean FVC increased from $73.6 \%$ (SD 16.9) of predicted value at baseline to $79.5 \%$ (SD 16.9) at 1 year and $80.6 \%$ (SD 19.1) at 2 years ( $n=37, P<0.001)$; the mean DLCO was $60.2 \%$ (SD 19.3) at baseline, 59.7\% (SD 17.7) at 1 year and $60.4 \%(S D 19.1)$ at 2 years $(n=35)$. The sHAQ score was available in 15 patients and decreased significantly from 0.96 (SD 0.8) at baseline to 0.73 (SD 0.6) at 1 year and 0.70 (SD $0.6)$ at 2 years $(P<0.001)$. The mean erythrocyte sedimentation rate decreased from $23.2 \mathrm{~mm} / \mathrm{h}$ (SD 19.8) at baseline to $16.1 \mathrm{~mm} / \mathrm{h}(\mathrm{SD} 11.3)$ at 1 year and $18.7 \mathrm{~mm} / \mathrm{h}$ (SD 23.6) at 2 years $(n=38, P<0.001)$. Anti-Scl-70 and anti-centromere autoantibodies did not change after autologous HSCT.

\section{Safety}

The NRM rate at 100 days and 2 years was $6.25 \%(95 \%$ CI 2.3-13) (Figure 1). Four of the five deaths not due to 


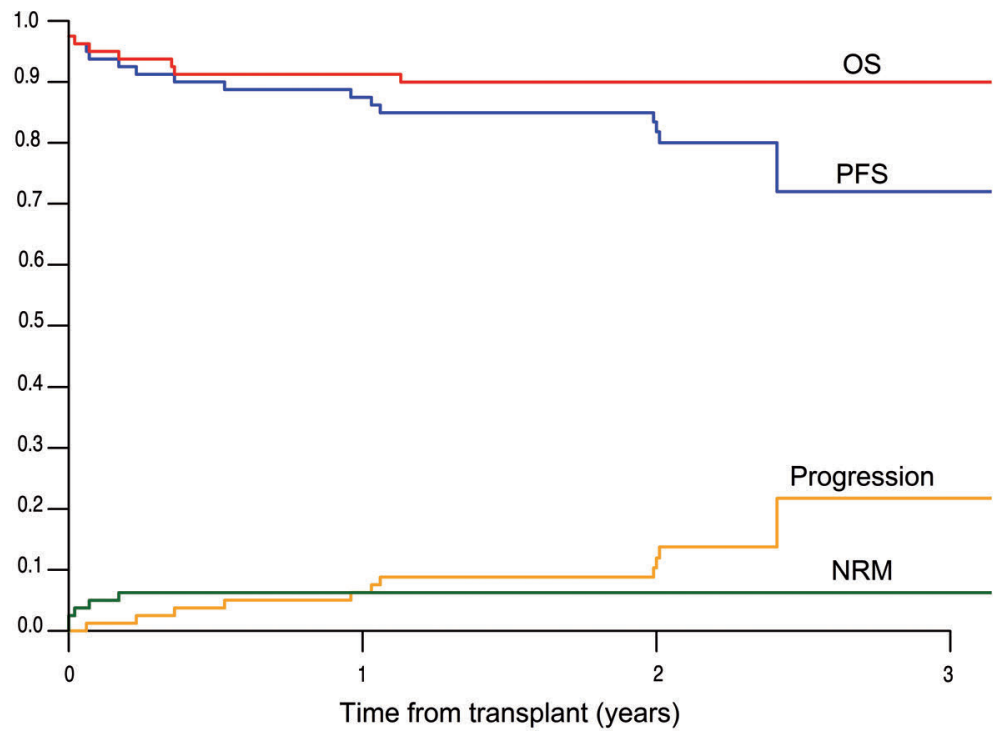

Figure 1. Kaplan-Meier curves of outcomes of patients undergoing autologous hematopoietic stem cell transplantation for severe systemic sclerosis. Overall survival (OS), progression-free survival (PFS), progression and non-relapse (treatment)related mortality (NRM) over time.

A

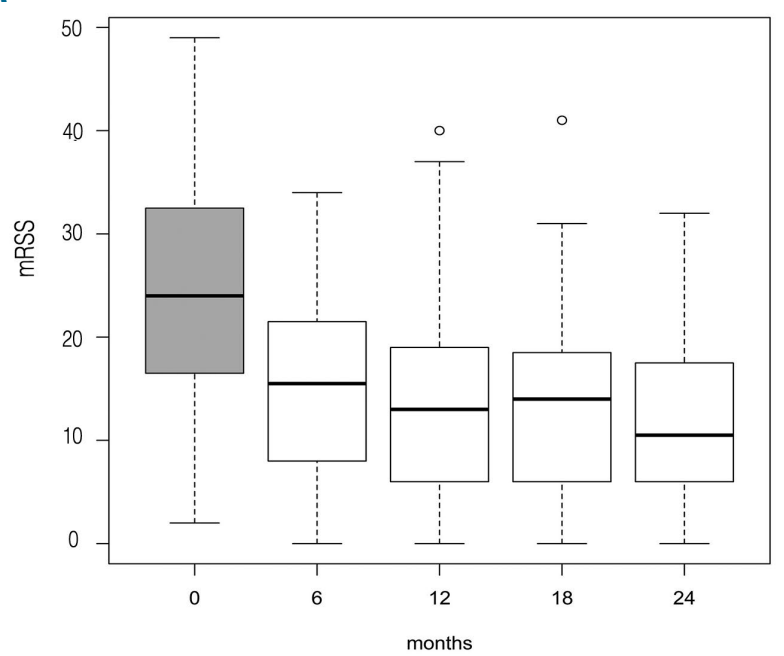

C

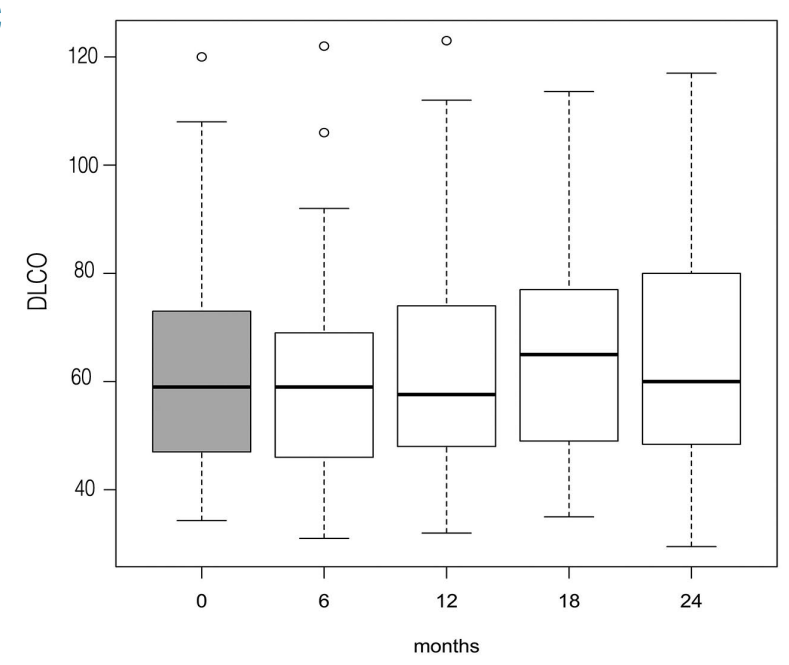

B

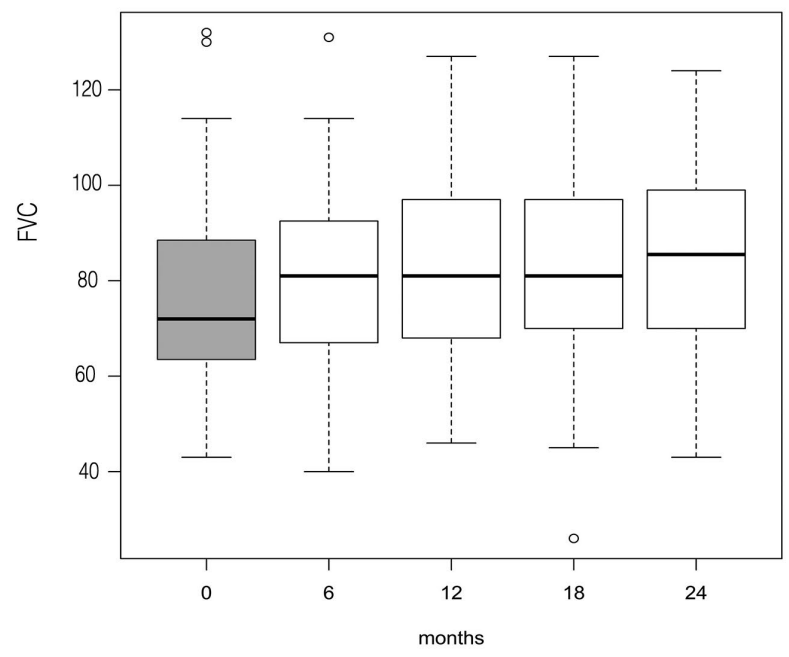

D

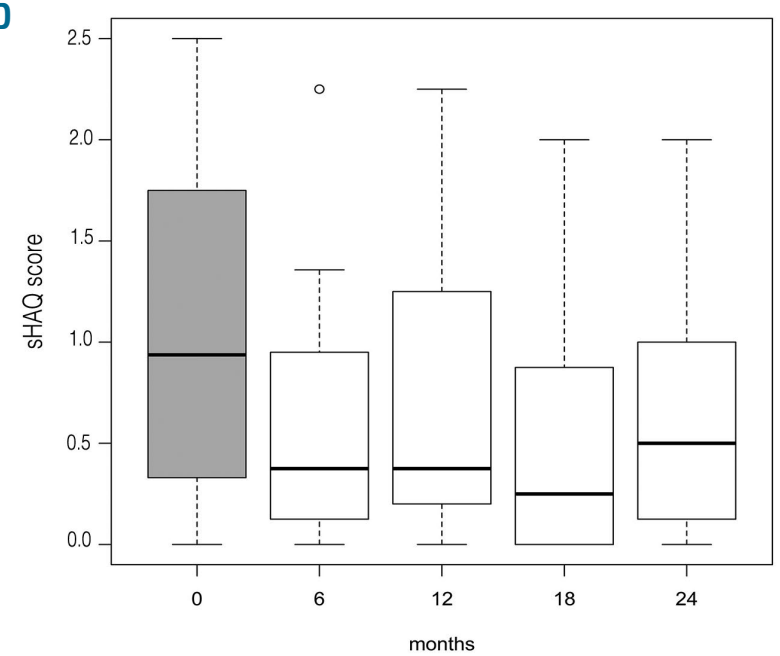

Figure 2. Boxplots of the four most important efficacy parameters in the $\mathbf{2 4}$ months following autologous hematopoietic stem cell transplantation for severe systemic sclerosis. (A) Improvement of the modified Rodnan skin score (mRSS) ( $n=55 ; P<0.001)$. (B) Improvement of forced vital capacity (FVC) ( $n=37$; $P=0.001$. (C) Stabilization of diffusion capacity of carbon monoxide (DLCO) ( $\mathrm{n}=35$; $P=0.01$ ). (D) Improvement of Scleroderma Health Assessment Questionnaire (sHAQ) score $(\mathrm{n}=15, P=0.001)$ 
Table 3. Baseline characteristics and results from the pretransplant evaluation in patients who died during the follow-up.

\begin{tabular}{|c|c|c|c|c|c|c|c|c|c|c|c|c|c|c|}
\hline $\begin{array}{l}\text { Age at } \\
\text { aliSCT/ } \\
\text { gender }\end{array}$ & $\begin{array}{l}\text { SAP/DBP } \\
\text { (HR) }\end{array}$ & $\begin{array}{l}\text { Resting } \\
\text { ECG }\end{array}$ & $\begin{array}{l}\text { 24h-Holter } \\
\text { ECG }\end{array}$ & $\begin{array}{l}\text { Echo } \\
\text { LVEF } \\
(\%)\end{array}$ & $\begin{array}{l}\text { Echo } \\
\text { PASP }\end{array}$ & $\begin{array}{l}\text { Cardiac } \\
\text { MRl }\end{array}$ & RHC & Proteins & Haematuria & $\begin{array}{l}\text { Serum } \\
\text { creatinine } \\
\text { (umol/L) }\end{array}$ & $\begin{array}{l}\text { Mobilization } \\
\text { CYC } \\
\left(\mathrm{g} / \mathrm{m}^{2}\right)\end{array}$ & $\begin{array}{l}\text { Conditioning } \\
\text { regimen } \\
\text { (mg/kg) }\end{array}$ & $\begin{array}{l}\text { Time to } \\
\text { death } \\
\text { (days) }\end{array}$ & Cause of death \\
\hline $28 / \mathrm{F}$ & $90 / 60(80)$ & Normal & Normal & 60 & 19 & Normal & Not done & Positive & Positive & 36 & 2 & CYC 200 & 1 & $\begin{array}{l}\text { aHSCT related: cardiac } \\
\text { toxicity }\end{array}$ \\
\hline $46 / \mathrm{F}$ & $110 / 75(90)$ & Normal & Not done & 67 & 23 & Normal & Not done & Negative & Negative & 48 & 2 & CYC 200 & 1 & $\begin{array}{l}\text { aHSCT related: cardiac } \\
\text { toxicity and CYC } \\
\text { toxicity }\end{array}$ \\
\hline $55 / \mathrm{F}$ & $125 / 75(65)$ & Normal & Abnormal & 68 & 31 & Normal & Not done & Negative & Negative & 56 & 3 & CYC 200 & 9 & $\begin{array}{l}\text { aHSCT related: cardiac } \\
\text { toxicity and CYC } \\
\text { toxicity }\end{array}$ \\
\hline $35 / \mathrm{F}$ & $100 / 70(88)$ & Normal & Not done & 62 & & Not done & Not done & Negative & Negative & 35 & 2 & CYC 200 & 61 & $\begin{array}{l}\text { aHSCT related: cardiac } \\
\text { toxicity and CYC } \\
\text { toxicity }\end{array}$ \\
\hline $52 / \mathrm{M}$ & $135 / 85(72)$ & Normal & Abnormal & 55 & 20 & Abnormal & $\begin{array}{c}\text { Mean } \\
\text { PAP=17 } \\
\text { No fluid } \\
\text { challenge }\end{array}$ & Positive & Negative & 62 & 3 & $\begin{array}{l}\text { CYC } 100+ \\
\text { Thiotepa } 10\end{array}$ & 26 & $\begin{array}{l}\text { aHSCT related: infection } \\
+\mathrm{MOF}\end{array}$ \\
\hline $58 / \mathrm{M}$ & $120 / 80(82)$ & Normal & Normal & 66 & 30 & Normal & Not done & Negative & Negative & 115 & 3 & CYC 200 & 128 & $\begin{array}{l}\text { Progression: } \\
\text { Pulmonary toxicity }\end{array}$ \\
\hline $43 / \mathrm{M}$ & $120 / 80(100)$ & Normal & Normal & 64 & 16 & Normal & Not done & Positive & Positive & 35 & 4 & CYC 200 & 131 & Progression \\
\hline $51 / \mathrm{F}$ & $120 / 70(70)$ & Normal & Normal & 72 & 30 & Normal & Not done & Negative & Negative & 37 & 3 & CYC 200 & 414 & Progression \\
\hline
\end{tabular}

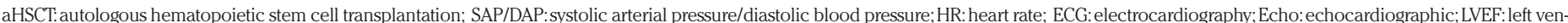

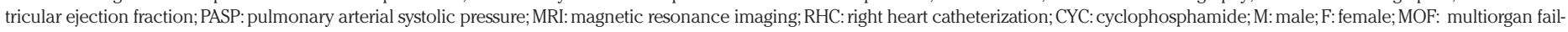
ure; NA: not applicable;

relapse were related to a cardiac event, due to cyclophosphamide toxicity in three patients. A 35-year old female, who died from cardiac toxicity and multiorgan failure on day +61 after autologous HSCT, had normal resting ECG and LVEF (62\%) on echocardiography, and abnormal LVEF on heart scintigraphy (47\%); MRI and RHC were not performed at baseline. Three other patients died from cardiac toxicity at day $+1(n=2)$ and day $+9(n=1)$, with normal cardiac MRI and no RHC evaluation at baseline. A fifth patient (conditioning with thiotepa + reduced-dose cyclophosphamide) died from cytomegalovirus reactivation with viral copies detectable also in the lung, with bacterial (Pseudomonas) pneumonia and multiorgan failure at day +21 . This patient had a normal resting ECG, with an abnormal $24 \mathrm{~h}$ ECG, abnormal MRI and a mean pulmonary artery pressure of $17 \mathrm{mmHg}$ on RHC performed without fluid challenge at baseline. During the 24 months of follow-up, three additional patients died, all due to disease progression. Baseline characteristics of all patients who died during the follow-up and the respective causes of death are presented in Table 3 .

During the 100 days after transplantation, a total of 119 adverse events were reported among the 80 patients as detailed in Table 4. The percentage of infectious complications was not significantly different between the patients who did or did not receive CD34+-selected grafts $(P=0.44)$, or according to the cyclophosphamide dose at the time of mobilization ( $2 \mathrm{~g} / \mathrm{m}^{2}$ vs. $4 \mathrm{~g} / \mathrm{m}^{2}, P=0.16$ ) or depending on whether they were or were not given G-CSF after their transplant $(P=0.32)$ (Online Supplementary Table S3).

\section{Discussion}

The NISCC1 study is the largest prospective cohort today analyzing the efficacy and safety of autologous
HSCT in 80 patients with severe SSc in a real-life setting. The PFS rate was $82 \%$ and significant improvements were observed in skin score, lung function and quality of life during the 2 years of follow-up, consistent with results of previous randomized controlled trials ${ }^{14-16}$ and earlier studies. ${ }^{11-13}$ Nevertheless, the NRM rate associated with autologous HSCT was $6.25 \%$, with most (4 out of 5 ) of the deaths being related to early cardiac events; this is similar to the $6 \%$ NRM rate reported retrospectively among 89 SSc patients transplanted between 2002 to 2011 in one USA and one Brazilian expert center using a non-myeloablative conditioning regimen. ${ }^{21}$

We used PFS as the primary endpoint to assess autologous HSCT efficacy rather than event-free survival, as previously used ${ }^{14,15}$ and which was defined as survival without respiratory, renal, or cardiac failure. PFS, which encompasses disease progression and toxic deaths, better reflects safety and efficacy of HSCT.

In NISSC1, the incidence of progression was $6.3 \%$ and $11.9 \%$ at 1 and 2 years, respectively, highlighting the need to identify patients who may benefit from early maintenance therapy after autologous HSCT. Several mechanisms contribute to the resetting of the immunological response and disease control after autologous HSCT, and vary according to individual patients. ${ }^{12} \mathrm{We}$ showed that the immune reconstitution profile and $\mathrm{T}$ cell repertoire diversity together with a decrease in $\mathrm{mRSS}>25 \%$ and in FVC $>10 \%$ at $1-2$ years after transplantation could identify long-term responders and nonresponders/relapsing patients at 4-5 years. ${ }^{22}$ The immune reconstitution process and the balance between reinduction of tolerance and sustained autoreactivity after transplantation will depend on the type of conditioning regimen used to ablate the original immune system, the effect of ATG or ex vivo CD34+-cell selection to 
further deplete/eliminate residual autoreactive cells and the use of post-transplant maintenance immunosuppression. . $^{15,16,22-24}$

All patients in the NISCC1 study received a 'non-myeloablative' cyclophosphamide-based conditioning regimen, which included rabbit-derived ATG in all cases. The degree of $\mathrm{T}$-cell depletion for each patient varied according to the type and total dose of ATG as well as timing of its administration in relation to the peripheral blood stem cell infusion, and whether the infused cells had undergone $e x$ vivo $\mathrm{CD} 34^{+}$selection. In a previous retrospective EBMT analysis, we failed to demonstrate a benefit of ex vivo $\mathrm{CD}^{+} 4^{+}$-cell selection ${ }^{23}$ in $138 \mathrm{SSc}$ patients with similar baseline characteristics transplanted between 2000 and 2012. Surprisingly, the NISCC1 study showed a superior response to treatment in patients who received a $\mathrm{CD} 34^{+}-$ selected graft, with no increased rate of infectious complications, consistent with the recent findings of Ayano et al. in a retrospective analysis of $19 \mathrm{SSc}$ patients. ${ }^{24}$ The reason for the difference in outcomes between these studies is unclear, but the population included in the NISCC1 prospective cohort was more homogenous in terms of conditioning, including in vivo T-cell depletion with rabbitderived ATG. All patients in the NISCC1 study received rabbit ATG formulations (although of different brands) while, in the EBMT retrospective study ${ }^{23} 64 \%$ of patients treated with ATG received a rabbit-derived product and $35 \%$ the horse-derived ATG formulation, which were shown to be associated with significantly different clinical outcomes in aplastic anemia. ${ }^{25}$ This important finding raises the question of whether a randomized, controlled trial should be performed to clarify whether more profound depletion of autoreactive cells from the peripheral blood stem cell graft by $\mathrm{CD} 34^{+}$-cell selection may translate into sustained clinical benefit in SSc patients after autologous HSCT, without adversely affecting safety and cost-effectiveness.

During the immune reconstitution process, the re-emergence of naive $T$ and $B$ cells, the renewal of the immune repertoire and reinstatement of synergistic immunoregulatory mechanisms are expected. Therefore maintenance or rapid reintroduction of immunosuppression after autologous HSCT may improve patients' outcome. The ongoing open-label trial (ClinicalTrials.gov identifier: NCT01413100) analyzing the addition of mycophenolate mofetil for 2 years after autologous HSCT and the next NISCC2 study (ClinicalTrials.gov identifier: NCT 03444805) analyzing posttransplantation management may clarify the potential benefit of post-transplant maintenance immunosuppression.

A critical point for autologous HSCT in autoimmune diseases is safety. The risks of early transplant-related complications and mortality vary according to the type of disease, pre-existing internal organ involvement, the experience of the transplant center and the intensity of the conditioning regimen. ${ }^{12,26,27}$ Major cardiac adverse events and NRM after autologous HSCT in SSc are predominantly related to primary cardiac and lung involvement, which are underevaluated in the absence of systematic MRI and RHC at baseline. ${ }^{28,29}$ Our study revealed important variations in clinical practice in the pre-transplant cardio-pulmonary evaluation process. Although all patients who died during the first 100 days had normal resting ECG and LVEF on echocardiography at baseline, they were not all assessed by $24 \mathrm{~h}$ ECG (3/5), MRI (4/5) or RHC with fluid challenge (1/5). Overall, $24 \mathrm{~h}$ ECG was only used in 75\% of the NISCC1 patients to detect subclinical rhythm or conduction disturbances at baseline, although it has been recommended since 2004. ${ }^{27}$ Similarly, cardiac MRI and RHC with fluid challenge were only performed in $74 \%$ and $21 \%$ of the patients, respectively, while their use has been advocated among expert centers since 2012 to mini-

Table 4. Complications reported in the 100 days following autologous hematopoietic stem cell transplantation among the 80 patients treated for severe systemic sclerosis.

\begin{tabular}{lc}
\hline Post-transplant non-infectious complications in 21 (26\%) patients & $\begin{array}{c}\text { Events } \\
(\mathbf{n = 2 4})\end{array}$ \\
\hline CYC-related acute cardiomyopathy / 4 deaths & 4 \\
Cardiomyopathy with myocardial infarction & 1 \\
\hline ARDS + acute heart failure & 1 \\
Arrhythmias & 2 \\
\hline Pericardial effusion & 1 \\
Atrial flutter/fibrillation & 2 \\
\hline Allergy to ATG with respiratory failure + pulmonary hemorrhagic syndrome & 1 \\
ATG-related fever & 1 \\
\hline Acute pulmonary edema & 1 \\
Renal failure & 3 \\
\hline Psychosis/depression & 2 \\
Epistaxis & 1 \\
\hline Oral mucositis & 1 \\
Anal fissure & 1 \\
\hline Hemorrhagic cystitis & 2
\end{tabular}

\begin{tabular}{ll|} 
Post-transplant infections reported in $60(75 \%)$ patients & Events \\
$(n=95)$
\end{tabular}

Neutropenic fever with no documented infection 25

Catheter infection

2

Sepsis, including 1 septic shock

Sinusitis

Pneumonia

Endocarditis

Bacterial

Pneumonia

Pleuritis

Sepsis Gram-positive bacillus

Sepsis Gram-negative bacillus

Catheter infection not documented

Cellulitis

Viral

CMV reactivation

EBV reactivation

Pneumonia

Herpes simplex infection

BK virus cystitis

Hemorrhagic cystitis, adenovirus-positive

Fungal

Fungal sinus infection

Skin infection-right foot--Fusarium spp-

Candida stomatitis

Parasitic

Toxoplasma reactivation

CYC: cyclophosphamide; ARDS: acute respiratory distress syndrome; ATG: antithymocyte globulins; CMV: cytomegalovirus; EBV: Epstein-Barr virus. 
mize the risk of the transplant procedure..$^{21,30,31}$ From 2013 to 2017, the EBMT and collaborative partners worked collectively to establish common standards for the use and interpretation of cardiac MRI results and RHC with fluid challenge for a rigorous cardio-pulmonary assessment of SSc patients, carefully defining eligibility and selection criteria for transplant referral. ${ }^{32}$

Optimized treatment regimens are warranted to improve the safety of autologous HSCT, even in patients without cardiac involvement. Interestingly, the NRM rate in the NISCC1 study was similar to the $6 \%$ reported among 32 SSc patients transplanted between 2005 and 2011, using a 'myeloablative' conditioning regimen comprising total body irradiation (8 Gy), cyclophosphamide $(120 \mathrm{mg} / \mathrm{kg})$ and horse-derived ATG in the prospective SCOT randomized controlled trial. ${ }^{16}$ However, in the SCOT trial, NRM was primarily related to late fatal myelodysplastic syndromes, while no cyclophosphamide toxicity was reported. Within the follow-up period of the NISSC1 study, there were no reports of myelodysplastic syndrome or other myeloid malignancies. In addition, there were no reports of serious late infectious complications or secondary autoimmune phenomena that may occasionally present as long-term complications following autologous HSCT in autoimmune diseases. Although further follow-up is required, it is possible that there are long-term advantages, in terms of late effects, of the 'non-myeloablative' regimen used in the NISCC1 study.

Early toxicity remains the principal challenge, including a NRM rate of $6 \%$. To reduce toxicity and thus treatmentrelated mortality of the conditioning, a more individualized regimen with respect to heart, lung or kidney involvement might further improve patients' safety, without excluding patients with organ manifestations. Two studies addressing patients with heart involvement are ongoing, both using a conditioning regimen with a reduced dose of cyclophosphamide and the addition of either thiotepa, as in four patients in our NISCC1 cohort (NCT01895244), or rituximab and fludarabine followed by reinfusion of autologous hematopoietic stem cells (NCT03593902), although the hematopoietic recovery from this reduced-dose regimen is prompt and reinfusion is not necessary in this setting.

Because mobilization with G-CSF alone may exacerbate autoimmune diseases, there is a clinical rationale for combining G-CSF with cyclophosphamide. The use of $2 \mathrm{~g} / \mathrm{m}^{2}$ cyclophosphamide for mobilization was as effective as 4 $\mathrm{g} / \mathrm{m}^{2}$ at achieving the EBMT recommended minimum CD34+-cell yield, supporting dose reduction in the mobilization regimen to limit early toxicity as well as long-term risks associated with cumulative doses of cyclophosphamide. During the autologous HSCT procedures, inevitable infectious and non-infectious toxicity occurred especially following the high-dose cytotoxic conditioning and the cytopenic phase. A correlation between the num- ber of infused CD34 ${ }^{+}$cells and a reduced duration of aplasia after autologous HSCT has been described previous$1 y,{ }^{33,34}$ which was confirmed in the NISCC1 study, although $\mathrm{CD}^{+} 4^{+}$-cell dose and addition of G-CSF until recovery from aplasia were not associated with a reduced number of infectious complications.

The NISCC1 study has several limitations. Since the study was a non-interventional trial, follow-up physical and laboratory evaluations, although recommended by EBMT guidelines, ${ }^{17}$ were not mandatory, and were not obtained at all time-points. The non-interventional study design was also the cause of significant variation in data collection practices by the 13 participating sites. Data were prospectively collected and checked for consistency by the EBMT study office; however, when some examinations were not performed, as may occur in routine clinical practice, data were classified as "missing". The NISCC1 results showed significant variation in local practices when evaluating patients at baseline. Similarly, the number of reported days for mobilization varied largely according to centers; indeed, some patients were hospitalized for the whole duration of this procedure, whereas in other cases it was carried in part on an outpatient basis.

We aimed to investigate the factors influencing the safety and efficacy of autologous HSCT. We used validated parameters to assess post-transplant outcome, such as mRSS or lung function tests despite their large intra- and inter-individual variability. The results enable deeper analysis of the benefits and risks of the transplant procedure in relation to both SSc-related factors, including the extent of heart, lung and kidney involvement at the time of patient referral, and transplant-related factors such as the conditioning regimen and graft manipulation. No international consensus has yet validated a unique activity score in SSc and further studies will be necessary in the post-transplant setting to investigate disease activity, the concept of drug-free remission and longer term outcomes, with or without post-transplant interventions.

In conclusion, the NISSC1 is a large prospective real-world data study of importance for patients, clinicians, and healthcare payers, given the poor prognosis and burden of disability of patients with severe SSc. It allowed us to assess multicenter adherence and compliance to good clinical practice and adds significantly to the evidence base supporting the delivery of autologous HSCT for severe SSc in routine clinical practice.

\section{Disclosures}

No conflicts of interests to diclose.

\section{Contributions}

$J H, D F, M B, J S$ and $M L$ : conception, study design, analyses and interpretation of data. MO, HUS, NDP, TD, MS, RS, $T M, G P, B S, D M, E M, B L, J O B, M S, A H, F O, L K$ and $Z M$ : acquisition of data.

\section{References}

1. Denton CP, Khanna D. Systemic sclerosis. Lancet. 2017;390(10103):1685-1699.

2. Elhai M, Meune C, Boubaya $M$, et al. Mapping and predicting mortality from systemic sclerosis. Ann Rheum Dis.
2017;76(11):1897-1905

3. Herrick AL, Peytrignet S, Lunt $M$, et al. Patterns and predictors of skin score change in early diffuse systemic sclerosis from the European Scleroderma Observational Study. Ann Rheum Dis. 2018;77(4):563-570.

4. Fransen J, Popa-Diaconu D, Hesselstrand R, et al. Clinical prediction of 5 -year survival in systemic sclerosis: validation of a simple prognostic model in EUSTAR centres. Ann Rheum Dis. 2011;70(10):1788-1792.

5. Tyndall AJ, Bannert B, Vonk M, et al. Causes and risk factors for death in systemic sclerosis: a study from the EULAR Scleroderma Trials and Research (EUSTAR) database. Ann Rheum Dis. 2010;69(10):1809-1815. 
6. Hao Y, Hudson M, Baron M, et al. Early mortality in a multinational systemic sclerosis inception cohort. Arthritis Rheumatol. 2017;69(5):1067-1077.

7. Steen VD, Medsger TA Jr, Rodnan GP. Dpenicillamine therapy in progressive systemic sclerosis (scleroderma): a retrospective analysis. Ann Intern Med. 1982;97(5):652-659.

8. Steen VD, Medsger TA, Jr. The value of the Health Assessment Questionnaire and special patient-generated scales to demonstrate change in systemic sclerosis patients over time. Arthritis Rheum. 1997;40(11):19841991.

9. Herrick AL, Pan X, Peytrignet S, et al. Treatment outcome in early diffuse cutaneous systemic sclerosis: the European Scleroderma Observational Study (ESOS). Ann Rheum Dis. 2017;76(7):1207-1218.

10. Kowal-Bielecka O, Fransen J, Avouac J, et al. Update of EULAR recommendations for the treatment of systemic sclerosis. Ann Rheum Dis. 2017;76(8):1327-1339

11. Farge D, Marolleau JP, Zohar S, et al. Autologous bone marrow transplantation in the treatment of refractory systemic sclerosis: early results from a French multicentre phase I-II study. Br J Haematol. 2002;119 (3):726-739.

12. Farge D, Labopin M, Tyndall A, et al. Autologous hematopoietic stem cell transplantation for autoimmune diseases: an observational study on 12 years' experience from the European Group for Blood and Marrow Transplantation Working Party on Autoimmune Diseases. Haematologica. 2010;95(2):284-292.

13. Henes JC, Schmalzing M, Vogel W, et al. Optimization of autologous stem cell transplantation for systemic sclerosis -- a singlecenter longterm experience in 26 patients with severe organ manifestations. J Rheumatol. 2012;39(2):269-275.

14. Burt RK, Shah SJ, Dill K, et al. Autologous non-myeloablative haemopoietic stem-cell transplantation compared with pulse cyclophosphamide once per month for systemic sclerosis (ASSIST): an open-label, randomised phase 2 trial. Lancet. 2011;378 (9790):498-506.

15. van Laar JM, Farge D, Sont JK, et al. Autologous hematopoietic stem cell transplantation vs intravenous pulse cyclophosphamide in diffuse cutaneous systemic sclerosis: a randomized clinical trial. JAMA. 2014;311(24):2490-2498.
16. Sullivan KM, Goldmuntz EA, Keyes-Elstein $\mathrm{L}$, et al. Myeloablative autologous stem-cell transplantation for severe scleroderma. N Engl J Med. 2018;378(1):35-47.

17. Snowden JA, Saccardi R, Allez M, et al. Haematopoietic SCT in severe autoimmune diseases: updated guidelines of the European Group for Blood and Marrow Transplantation. Bone Marrow Transplant. 2012;47(6):770-790.

18. Duarte RF, Labopin M, Bader $\mathrm{P}$, et al Indications for haematopoietic stem cell transplantation for haematological diseases, solid tumours and immune disorders: current practice in Europe, 2019. Bone Marrow Transplant. 2019;54(10):1525-1552.

19. Sullivan KM, Majhail NS, Bredeson C, et al. Systemic sclerosis as an indication for autologous hematopoietic cell transplantation: position statement from the american society for blood and marrow transplantation. Biol Blood Marrow Transplant. 2018;24(10): 1961-1964.

20. Passweg JR, Baldomero H, Bader P, et al. Is the use of unrelated donor transplantation leveling off in Europe? The 2016 European Society for Blood and Marrow Transplant activity survey report. Bone Marrow Transplant. 2018;53(9):1139-1148.

21. Burt RK, Oliveira MC, Shah SJ, et al Cardiac involvement and treatment-related mortality after non-myeloablative haemopoietic stem-cell transplantation with unselected autologous periphera blood for patients with systemic sclerosis: a retrospective analysis. Lancet. 2013;381 (9872):1116-1124

22. Farge D, Arruda LC, Brigant F, et al. Longterm immune reconstitution and $\mathrm{T}$ cell repertoire analysis after autologous hematopoietic stem cell transplantation in systemic sclerosis patients. J Hematol Oncol. 2017;10(1):21.

23. Oliveira MC, Labopin M, Henes J, et al. Does ex vivo CD34+ positive selection influence outcome after autologous hematopoietic stem cell transplantation in systemic sclerosis patients? Bone Marrow Transplant. 2016;51(4):501-505

24. Ayano M. Tsukamoto $\mathrm{H}$, Mitoma $\mathrm{H}$, et al. CD34-selected versus unmanipulated autologous haematopoietic stem cell transplantation in the treatment of severe systemic sclerosis: a post hoc analysis of a phase I/II clinical trial conducted in Japan. Arthritis Res Ther. 2019;21(1):30.
25. Young NS. Aplastic anemia. N Engl J Med. 2018;379(17):1643-1656.

26. Gratwohl A, Passweg J, Bocelli-Tyndall C, et al. Autologous hematopoietic stem cell transplantation for autoimmune diseases. Bone Marrow Transplant. 2005;35(9):869-879.

27. Saccardi R, Tyndall A, Coghlan G, et al. Consensus statement concerning cardiotoxicity occurring during haematopoietic stem cell transplantation in the treatment of autoimmune diseases, with special reference to systemic sclerosis and multiple sclerosis. Bone Marrow Transplant. 2004;34(10):877881.

28. Mousseaux E，Agoston-Coldea L Marjanovic Z, et al. Left ventricle replacement fibrosis detected by CMR associated with cardiovascular events in systemic sclerosis patients. J Am Coll Cardiol. 2018;71(6): 703-705.

29. Mueller KA, Mueller, II, Eppler D, et al Clinical and histopathological features of patients with systemic sclerosis undergoing endomyocardial biopsy. PLoS One. 2015;10(5):e0126707.

30. Burt RK, Shah SJ, Gheorghiade M Ruderman E, Schroeder J. Hematopoietic stem cell transplantation for systemic sclerosis: if you are confused, remember: "it is a matter of the heart". J Rheumatol 2012;39(2):206-209.

31. Krumm P, Mueller KA, Klingel $K$, et al. Cardiovascular magnetic resonance pattern of biopsy proven cardiac involvement in systemic sclerosis. J Cardiovasc Magn Reson. 2016;18(1):70

32. Farge D, Burt RK, Oliveira MC, et al. Cardiopulmonary assessment of patients with systemic sclerosis for hematopoietic stem cell transplantation: recommendation from the European Society for Blood and Marrow Transplantation Autoimmune Diseases Working Party and collaborating partners. Bone Marrow Transplant. 2017;52(11):1495-1503.

33. Keever-Taylor CA, Heimfeld S, Steinmiller $\mathrm{KC}$, et al. Manufacture of autologous CD34(+) selected grafts in the NIAID-sponsored HALT-MS and SCOT multicenter clinical trials for autoimmune diseases. Bio Blood Marrow Transplant. 2017;23(9):1463 1472.

34. McSweeney PA, Nash RA, Sullivan KM, et al. High-dose immunosuppressive therapy for severe systemic sclerosis: initial out comes. Blood. 2002;100(5):1602-1610. 